\title{
HERA INCLUSIVE NEUTRAL AND CHARGED CURRENT CROSS SECTIONS AND A NEW PDF FIT, HERAPDF 2.0*
}

\author{
ZHIQING ZHANG
}

Laboratoire de l'Accélérateur Linéaire, Univ. Paris-Sud et IN2P3/CNRS, France

(Received November 12, 2015)

In this paper, I present the brand new results from the H1 and ZEUS collaborations on the combination of all previously published inclusive deep inelastic cross sections at HERA for neutral and charged current $e^{ \pm} p$ scattering for zero beam polarisation and the corresponding parton distributions functions, HERAPDF 2.0, at up to next-to-next-to-leading order (NNLO). The results also include a new precise determination at next-to-leading order (NLO) of the strong coupling constant $\alpha_{\mathrm{s}}\left(M_{Z}^{2}\right)=$ $0.1184 \pm 0.0016$ (excluding scale uncertainties) based on a simultaneous fit to the combined inclusive cross section data and jet production data.

DOI:10.5506/APhysPolBSupp.8.957

PACS numbers: 13.60.-r, 13.60.Hb, 12.38.-t, 12.39.--x

\section{Introduction}

The $e^{ \pm} p$ collider, HERA, used to be the largest electron microscope of the world, where the inclusive deep inelastic scattering (DIS) of neutral and charged current (NC and $\mathrm{CC}$ ) interactions has been studied over an unprecedented large kinematic region of Bjorken $x$ and negative four-momentumtransfer squared $Q^{2}$.

Based on the combined inclusive NC and CC cross sections measured by the H1 and ZEUS experiments using data taken from 1992 to 2000 in phase 1 (HERA I), a set of parton distribution functions (PDFs), HERAPDF 1.0, was previously obtained [1]. These data have also been the primary input for all modern PDF sets such as CT10 [2], MMHT2014 [3], NNPDFs [4] and ABM11 [5].

In phase 2 (HERA II), from 2002 to 2007, the integrated luminosity of the $e^{+} p\left(e^{-} p\right)$ collision amounts to about $150 \mathrm{pb}^{-1}\left(235 \mathrm{pb}^{-1}\right)$, representing an increase by a factor of 1.5 (15) over that of the corresponding number

${ }^{*}$ Presented at EDS Blois 2015: The $16^{\text {th }}$ Conference on Elastic and Diffractive Scattering, Borgo, Corsica, France, June 29-July 4, 2015. 
at HERA I. In addition, the $e^{ \pm}$beams were longitudinally polarised at HERA II. However, for the derivation of HERAPDF 2.0, the HERA II data were corrected to zero beam polarisation so that a full combination with the unpolarised cross sections from HERA I could be made.

This write-up is organised as follows. In Sec. 2, the data combination is briefly described. In Sec. 3, the QCD analysis to extract PDFs from the combined inclusive data is presented, followed by a summary in Sec. 4. Full details of the analysis are given in [6].

\section{Data combination}

In total, 41 data sets have been used in the combination, of which 21 are from HERA I, taken mainly at nominal proton beam energy $\left(E_{p}\right)$ of $920 \mathrm{GeV}$ but also at $820 \mathrm{GeV}$, and 12 from HERA II are at $920 \mathrm{GeV}$ and 4 each at 575 and $460 \mathrm{GeV}$, corresponding to centre-of-mass energies $(\sqrt{s})$ of 320, 300, 251 and $225 \mathrm{GeV}$.

A total number of 2927 individual data points is transformed and combined into a set of 1307 common grid points. The transformation is performed based on predictions at high $Q^{2}$ using an in situ NLO QCD fit to the data and at low $Q^{2}$ a fractal model [7] based fit. The combination is made by averaging the transformed data points based on a $\chi^{2}$ minimisation method [8], assuming that there is only one correct value for the cross section of each process at each point of the phase space. The correlation within a data set and between different data sets is taken into account in the combination.

The combination has significantly improved not only the statistical precision but also the systematic one since H1 and ZEUS used different reconstruction methods. Moreover, for certain regions of the phase space, where one of the two experiments has superior precision compared to the other, the less precise measurement is fitted to the more precise measurement, with a simultaneous reduction of the correlated systematic uncertainty. This reduction propagates to the other points, including those which are based solely on the measurement from the less precise experiment. Two examples are shown in Fig. 1. The first example presents the reduction of the uncertainty from some of the selected individual $\mathrm{NC} e^{+} p$ cross sections to the combined one and the second one the reduction from the combined CC $e^{-} p$ HERA I data to the full combination. The first example also shows the strong scaling violation effect in the $\mathrm{NC}$ data which gives rise to the sensitivity to constrain the gluon distribution function of the proton. The data at different $E_{p}$ and thus $\sqrt{s}$ allow a direct measurement of the longitudinal structure function $F_{\mathrm{L}}$ providing further sensitivity to the gluon distributions. 

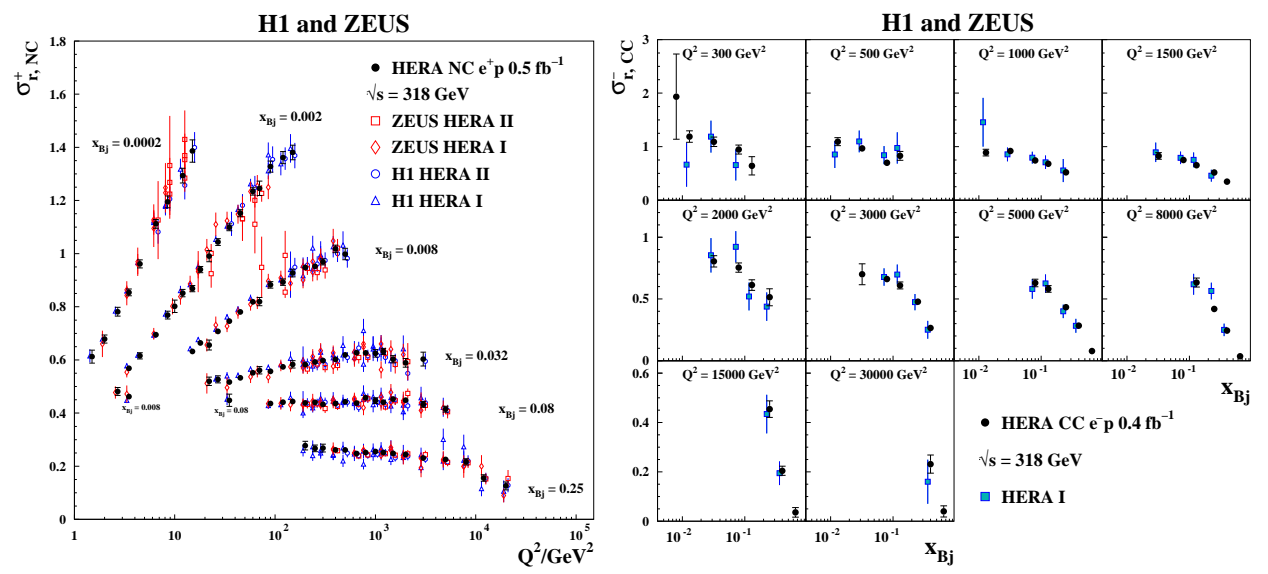

Fig. 1. Left: some of the selected $\mathrm{H} 1$ and ZEUS individual $\mathrm{NC} e^{+} p$ cross section measurements in HERA I and II in comparison with the corresponding combined data. Right: the combined $\mathrm{CC} e^{-} p$ cross sections from HERA I in comparison with the full combination.

\section{HERAPDF 2.0}

The availability of precision $\mathrm{NC}$ and $\mathrm{CC}$ cross sections over a large phase space allows HERAPDF to be based on $e p$ scattering data only and makes HERAPDF independent of any nuclear corrections. The new HERAPDF 2.0 is obtained following the same framework used for HERAPDF 1.0 [1]. Five PDFs $x u_{v}(x), x d_{v}(x), x \bar{U}(x), x \bar{D}(x)$ and $x g(x)$ are parameterised at an initial scale $\mu_{f_{0}}^{2}$, taken to be $1.9 \mathrm{GeV}^{2}$, in a generic form $x f(s)=A x^{B}(1-$ $x)^{C}\left(1+D x+E x^{2}\right)$ allowing $x g$ to have an extra term $-A_{g}^{\prime} x^{B_{g}^{\prime}}(1-x)^{C_{g}^{\prime}}$ for more flexibility [6]. The theoretical predictions of the $\mathrm{NC}$ and $\mathrm{CC}$ cross sections are obtained by convoluting the PDFs with coefficient functions at $\mathrm{LO}, \mathrm{NLO}$ or NNLO in the $\overline{\mathrm{MS}}$ scheme. The evolution in $Q^{2}$ of the PDFs is obtained from the DGLAP evolution equations [9]. The renormalisation and factorisation scales are chosen to be $\mu_{\mathrm{r}}^{2}=\mu_{\mathrm{f}}^{2}=Q^{2}$. The heavy quarks are treated in the general-mass variable-flavour-number scheme, RTOPT [10], for the NC predictions. For the CC predictions, the zero-mass approximation is used, since all HERA CC data are well beyond the $b$-quark mass scale.

Only cross section data above a minimum scale $Q_{\min }^{2}=3.5 \mathrm{GeV}^{2}$ are used in the fit. The fit is performed by minimising a $\chi^{2}$ function similar to HERAPDF 1.0 but with an additional logarithmic term introduced in [11]. The $\chi^{2}$ value per degree of freedom (d.o.f.) as a function of $Q_{\min }^{2}$ is shown in Fig. 2 (top left). The large $\chi^{2} /$ d.o.f. is investigated revealing tension between the QCD predictions and the data at both low and high $Q^{2}$. The HERAPDF 2.0 PDFs corresponding to choosing $Q_{\min }^{2}=3.5 \mathrm{GeV}^{2}$ are shown 

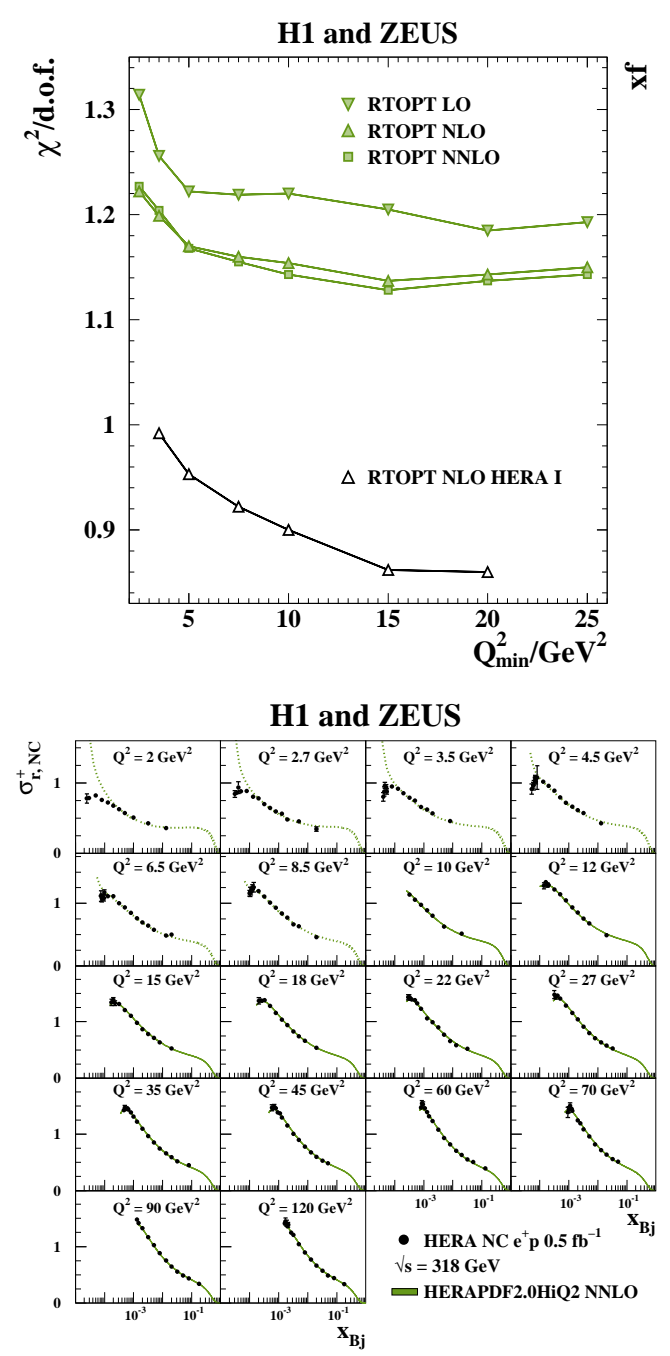

H1 and ZEUS

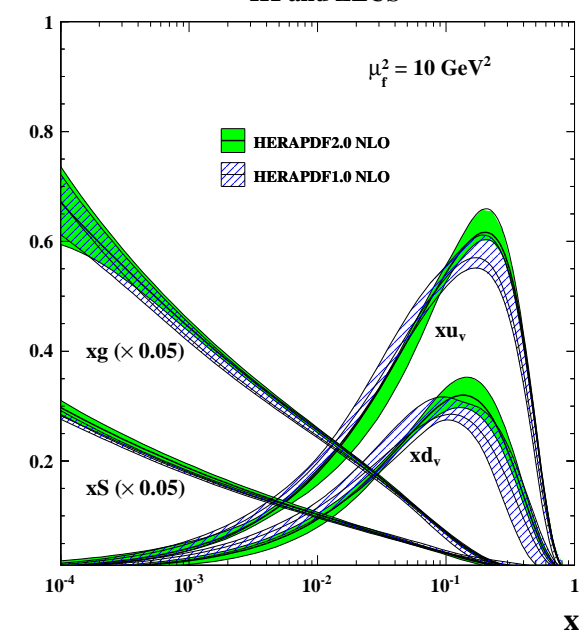

H1 and ZEUS

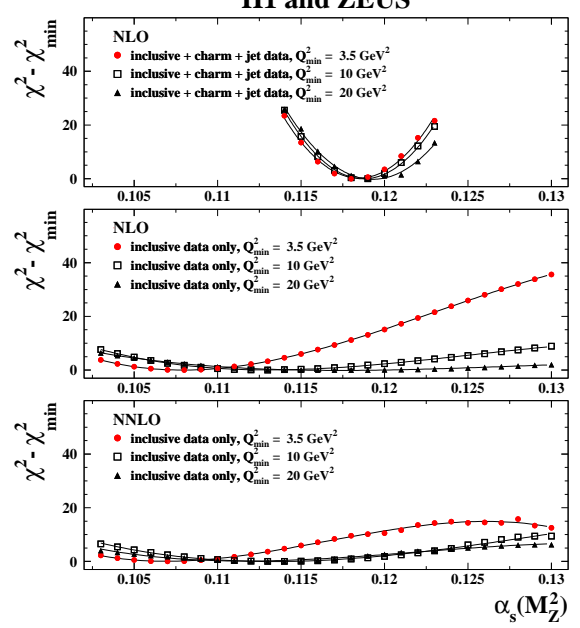

Fig. 2. Top left: the dependence of $\chi^{2}$ per degree of freedom (d.o.f.) on $Q_{\min }^{2}$ of LO, NLO and NNLO fits to the combined data. Top right: comparison of NLO PDFs $x u_{v}, x d_{v}, x S=2 x(\bar{U}+\bar{D})$ and $x g$ between HERAPDF 2.0 and 1.0. Bottom left: the combined low- $Q^{2} \mathrm{NC} e^{+} p$ cross section data in comparison with predictions from high $Q^{2}$ version of NNLO HERAPDF 2.0. Dotted lines indicate extrapolation into kinematic regions not included in the fit. Bottom right: $\chi^{2}-\chi_{\min }^{2}$ versus $\alpha_{\mathrm{s}}\left(M_{Z}^{2}\right)$ for fits with different $Q_{\min }^{2}$ using (upper part) inclusive charm and jet production at NLO, (middle part) inclusive $e p$ scattering data only at NLO and (lower part) inclusive $e p$ scattering data only at NNLO. 
for $\mu_{\mathrm{f}}^{2}=10 \mathrm{GeV}^{2}$ in Fig. 2 (top right) in comparison with that from HERAPDF 1.0. The new PDFs have substantially better precision in particular at high $x$ and the new $u$ and $d$ valence quarks are slightly harder but both sets are consistent within the uncertainty bands which include the experimental, model and parameterisation uncertainties [6].

Given the improved $\chi^{2}$ d.o.f. at higher $Q_{\min }^{2}$, a HERAPDF 2.0 variant with $Q_{\min }^{2}=10 \mathrm{GeV}^{2}$, HERAPDF $2.0 \mathrm{HIQ} 2$, is obtained, whose predictions at NNLO are compared with the combined NC $e^{+} p$ data for $Q^{2}$ below $120 \mathrm{GeV}^{2}$ in Fig. 2 (bottom left). Good agreement is observed in the fitted $Q^{2}$ region. The extrapolation to the low $Q^{2}$ and $x$ shows, however, clear deviation between the prediction and the low $x / Q^{2}$ data, which may indicate that one needs to add further contributions (e.g. resummation of $\ln (1 / x)$ terms) beyond the fixed NNLO order for the low $x / Q^{2}$ predictions.

The gluon distribution is known to be strongly correlated with the strong coupling constant $\alpha_{\mathrm{s}}$. Using the inclusive $\mathrm{NC}$ and $\mathrm{CC}$ cross section data is not able to simultaneously determine both. This is shown in Fig. 2 (bottom right). This is why the $\alpha_{\mathrm{s}}\left(M_{Z}^{2}\right)$ value is fixed for HERAPDF 2.0 to 0.118 at both NLO and NNLO and to 0.130 at LO. The same figure also shows that by adding jet data (combined charm data is also included, however its main effect is to constrain the pole mass of the charm quark to be $1.47(1.43) \mathrm{GeV}$ in the NLO (NNLO) fit), a simultaneous fit of $\alpha_{\mathrm{S}}\left(M_{Z}^{2}\right)$ and PDFs becomes possible. The resulting $\alpha_{\mathrm{S}}\left(M_{Z}^{2}\right)$ at NLO amounts to $0.1184 \pm 0.0009$ (exp.) \pm 0.0005 (model/parameterisation) \pm 0.0012 (hadronisation) ${ }_{-0.0030}^{+0.0037}$ (scale), which is competitive in precision with other NLO determinations. Note that the jet data cannot be included in an NNLO fit as its prediction in DIS has not been calculated to NNLO.

\section{Summary}

The full combination of the inclusive $\mathrm{NC}$ and $\mathrm{CC}$ cross sections published previously by H1 and ZEUS at HERA I and II is finally ready. The combined data, covering six orders of magnitude in both $x$ and $Q^{2}$ and reaching an unprecedented precision with typical values of below $0.5 \%$ for medium $Q^{2}$ region at the nominal centre-of-mass energy, represent a major legacy of HERA.

A new PDF set, HERAPDF 2.0 at LO, NLO and NNLO, is derived with a QCD fit to the combined inclusive data for $Q^{2}$ above $3.5 \mathrm{GeV}^{2}$. The new PDFs have smaller experimental uncertainties over that of HERAPDF 1.0.

By including additional jet and charmed data from HERA, a precision determination of $\alpha_{\mathrm{s}}\left(M_{Z}^{2}\right)=0.1184 \pm 0.0016$ at NLO (excluding the dominant scale uncertainties) in obtained. The value is in excellent agreement with the world average value of 0.1185 [12]. 


\section{REFERENCES}

[1] F.D. Aaron et al. [H1 and ZEUS collaborations], J. High Energy Phys. 1001, 109 (2010) [arXiv:0911.0884 [hep-ex]].

[2] H.L. Lai et al., Phys. Rev. D 82, 074024 (2010) [arXiv:1007.2241 [hep-ph]].

[3] L.A. Harland-Lang, A.D. Martin, P. Motylinski, R.S. Thorne, Eur. Phys. J. C 75, 204 (2015) [arXiv:1412.3989 [hep-ph]].

[4] R.D. Ball et al., Nucl. Phys. B 838, 136 (2010) [arXiv:1002.4407 [hep-ph]].

[5] S. Alekhin, J. Blumlein, S. Moch, Phys. Rev. D 86, 054009 (2012) [arXiv:1202.2281 [hep-ph]].

[6] H. Abramowicz et al. [H1 and ZEUS collaborations], arXiv:1506.06042 [hep-ex].

[7] T. Lastovicka, Eur. Phys. J. C 24, 529 (2002) [arXiv:hep-ph/0203260].

[8] F.D. Aaron et al. [H1 Collaboration], Eur. Phys. J. C 63, 625 (2009) [arXiv:0904.0929 [hep-ex]].

[9] V.N. Gribov, L.N. Lipatov, Sov. J. Nucl. Phys. 15, 438 (1972); 15, 675 (1972); L.N. Lipatov, Sov. J. Nucl. Phys. 20, 94 (1975); Y.L. Dokshitzer, Sov. Phys. JETP 46, 641 (1977); G.Altarelli, G. Parisi, Nucl. Phys. B 126, 298 (1977).

[10] R.S. Thorne, R.G. Roberts, Phys. Rev. D 57, 6871 (1998) [arXiv:hep-ph/9709442]; R.S. Thorne, Phys. Rev. D 73, 054019 (2006) [arXiv:hep-ph/0601245]; 86, 074017 (2012) [arXiv:1201.6180 [hep-ph]].

[11] F. Aaron et al. [H1 Collaboration], J. High Energy Phys. 1209, 061 (2012) [arXiv:1206.7007 [hep-ex]].

[12] K.A. Olive et al. [Particle Data Group], Chin. Phys. C 38, 090001 (2014). 